\title{
Long-term response in high-risk CLL
}

Although chronic lymphocytic leukemia (CLL) is often an indolent disease, highrisk cases have a dismal prognosis. The needs of this group of patients prompted a phase II trial to assess their outcome after treatment with reduced-intensity allogenic stem cell transplantation (RI allo-SCT).

\section{6 ...our approach is effective in} patients with del $17 \mathrm{p}-\ldots$ and its efficacy largely relies on graft-versus-leukemia activity 77

A total of 90 patients with high-risk CLL-as defined by relapse after therapy or progressive disease in the presence of adverse genetic indicators-received RI allo-SCT. Despite the high-risk cohort, the overall response rate was $94 \%$ with a relapse rate at 4 years of $40 \%$. Event-free survival was similar in all genetic subsets, including 17p deletions (del 17p-).

In 52 patients, the minimal residual disease was monitored. This assessment enabled the researchers, led by Peter Dreger, to carefully assess patient response. Dreger describes the most significant finding of this study as "that the RI allo-SCT can overcome the fatal prognosis of elderly patients with highrisk CLL at reduced risk compared with more aggressive SCT strategies." However, he believes the novel observations are that "our approach is effective in patients with del $17 \mathrm{p}-\ldots$ and its efficacy largely relies on graft-versus-leukemia activity."

Dreger plans three studies based on these data: a phase III trial comparing allo-SCT with non-transplant strategies, the development of a more targeted anti-leukimic effect and the addition of immunomodulators to RI allo-SCT.

\section{Rebecca Kirk}

Original article Dreger, P. et al. Allogenic stem cell transplantation provides durable disease control in poorrisk chronic lymphocytic leukemia: Iong-term clinical and MRD results of the German CLL Study Group CLL3X trial. Blood 116, 2438-2447 (2010) 